\title{
Repetitive Transient Ischaemic Attacks Secondary to a Floating Carotid Artery Thrombus in a Young Patient with Essential Thrombocythemia
}

\author{
Zoltán Bajkóno, ${ }^{1,2}$ Smaranda Maier ${ }^{1,2}$, Anca Moțățăianu ${ }^{1,2}$, Adina Stoian ${ }^{1,2}$, Laura Bărcuțean ${ }^{1,2}$, \\ Rodica Bălașa ${ }^{1,2}$ \\ ${ }^{1}$ University of Medicine and Pharmacy, Tîrgu Mureş, Romania \\ ${ }^{2}$ Department of Neurology, County Emergency Clinical Hospital, Tîrgu Mureș, Romania
}

\section{ABSTRACT}

A free-floating carotid artery thrombus is a very rare ultrasonographical finding in patients with acute ischemic cerebrovascular events. One of the main causes of this presentation is represented by the hypercoagulability status of the patients. We report the case of a young male patient who presented with several transient ischaemic attacks secondary to a mobile thrombus in the left carotid sinus. Two hematological abnormalities, essential thrombocytosis and methylene tetrahydrofolate reductase mutation were found to be related to the thrombus formation. The role of the latter in the pathogenesis of ischemic stroke is not well documented in the literature. Following antiplatelet and anticoagulant medication, there was a fast dissolution of the thrombus, followed by a favorable clinical outcome. Neurologists should be aware that young patients with stroke require extensive imagistic and laboratory screening for an accurate etiological diagnosis.

Keywords: repetitive transient ischemic attacks, carotid artery thrombus, essential thrombocytosis

\section{ARTICLE HISTORY}

Received: 5 June, 2015

Accepted: 20 June, 2015

\section{CORRESPONDENCE}

\section{Zoltán Bajkó}

Str. Gheorghe Marinescu nr. 50 540139 Tîrgu Mureș, Romania

Tel: +40 265215551

E-mail: bzoltan2003@yahoo.com

\section{INTRODUCTION}

A free-floating thrombus (FFT) in the carotid circulation is a very rare ultrasonographical finding and is usually diagnosed after an acute cerebrovascular event such as transient ischaemic attack or cerebral infarction, secondary to arterio-arterial embolism. The most frequent etiologies are a complicated atherosclerotic plaque, primary or secondary hypercoagulable states and cardioembolism. ${ }^{1,2}$
The case of a young patient with repeated transient ischaemic attacks and a mobile thrombus in the carotid artery secondary to hypercoagulability is reported. The subject and the institution agreed with presentation of these data. The procedures described were approved by the institution where the patient was treated. 

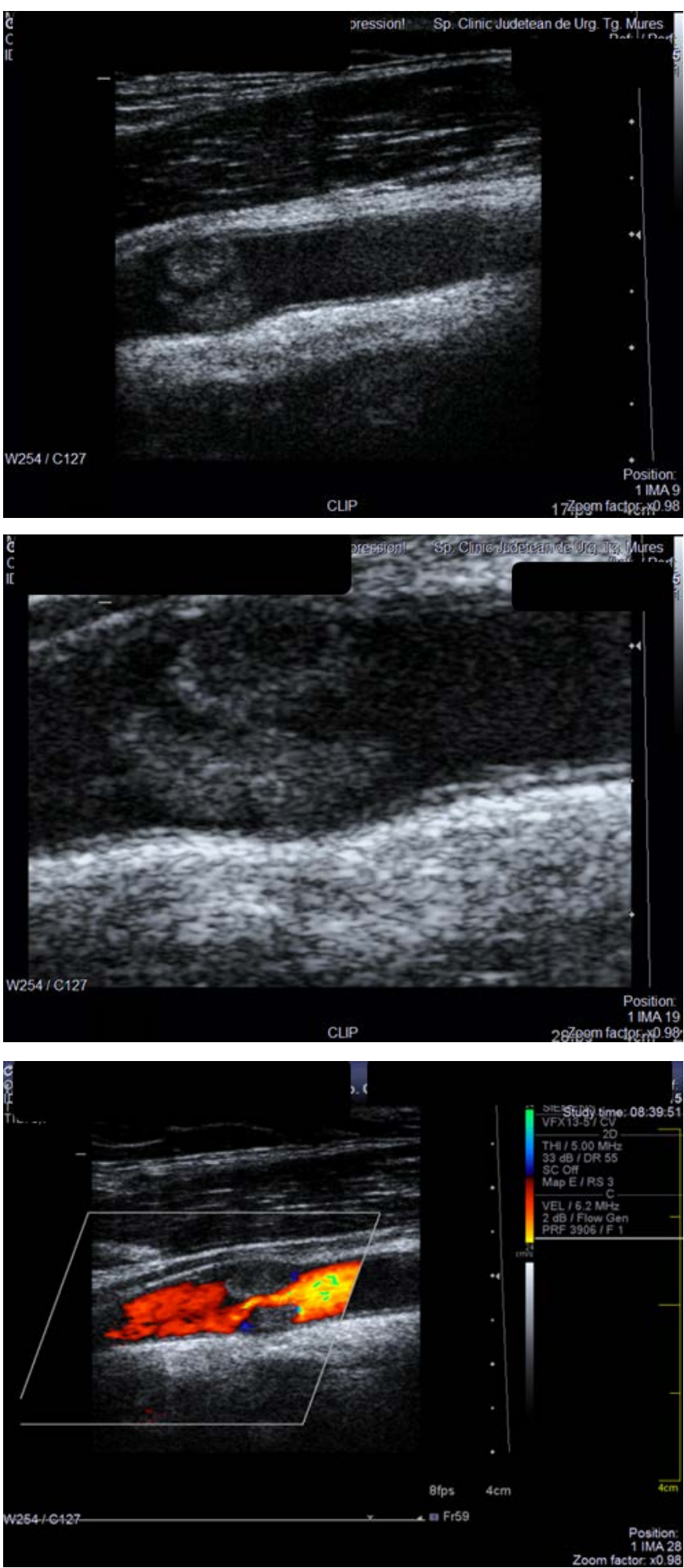

FIGURE 1. Carotid duplex ultrasound examination. Inhomogenous, mainly hypoechoic material is evidenced in the left carotid sinus (A, B - B mode examination) with significant luminal narrowing at this level (C - color mode examination).

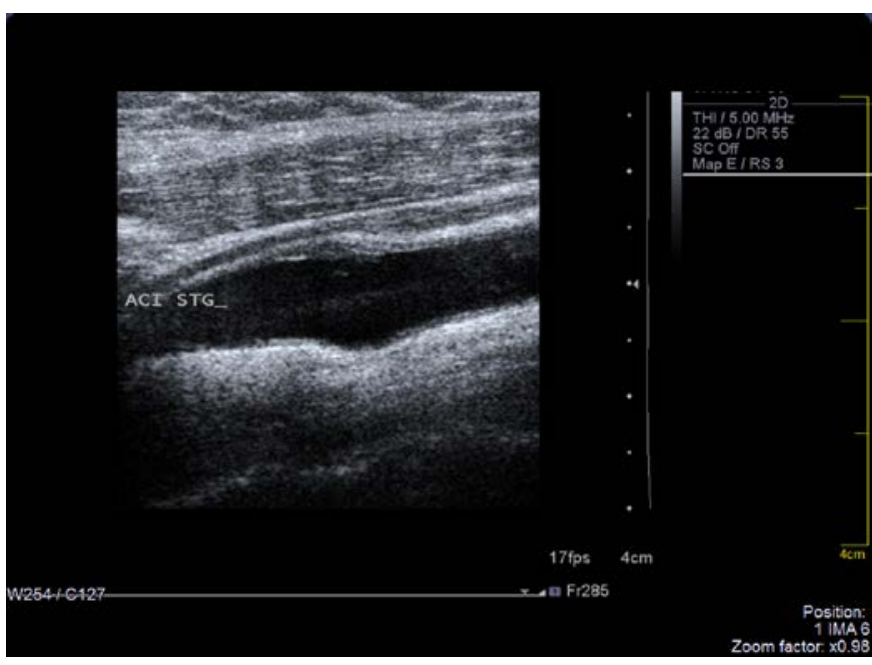

FIGURE 2. Control carotid ultrasound examination (B mode) 1 week after the initiation of anticoagulation treatment, revealing the disappearance of thrombus.

\section{CASE REPORT}

A 42-year-old male patient with mild obesity was admitted to a neurological ward following several short-lasting episodes of speech disturbance and right sided hemiparesis. Medical history and neurological examinations between attacks were normal. An emergency cerebral CT scan was performed, with nothing abnormal being observed. The cerebral magnetic resonance imaging (MRI) was also normal. Routine laboratory tests were normal, excepting a mild thrombocytosis $\left(551,000 / \mathrm{mm}^{3}\right)$. Carotid ultrasound examination revealed a mobile, hypoechoic material, suggestive of a thrombus, at the level of the left carotid sinus. There was significant stenosis at this level (Figure 1). No atherosclerotic changes were evidenced at the extracranial level, and transcranial color-coded duplex ultrasonography examination revealed normal flow velocities in the major cerebral arteries.

Thrombophilia examination evidenced a MTHFR A1298C mutation. The autoimmune and infectious markers were negative. Because of the thrombocytosis, the hematological consultant recommended further investigations to rule out the suspicion of essential thrombocytosis. Based on the consistently elevated platelet count, positive JAK2 mutation, the absence of BCR-ABL translocation between chromosomes 9 and 22, the absence of any causes for reactive thrombocytosis, the absence of peripheral blood evidence for a myelodysplastic syndrome (MDS), normal mean corpuscular volume (MCV) and low erythropoietin (EPO) level, the diagnosis of essential thrombocytosis (ET) was established. 
Taking into account the presence of a mobile thrombus with a high embolization potential, parenteral anticoagulation with low-molecular-weight heparin and antiplatelet medication with aspirin $75 \mathrm{mg}$ daily was initiated and continued for 7 days. After the initiation of anticoagulant therapy, the patient had no further transient ischemic attacks.

A control carotid ultrasound examination one week after the initiation of anticoagulant therapy showed that the thrombus had resolved (Figure 2).

The patient was discharged on oral anticoagulation (acenocumarol $4 \mathrm{mg}$ daily, with the control of the Internationalized Normal Ratio) and Aspirin 75 mg daily. For the hematological disorder, treatment with hydroxyurea was initiated and continued on long term.

During the 6-month follow-up period, the patient reported no further neurological complications.

\section{DISCUSSIONS}

A free-floating thrombus is defined as "an elongated thrombus attached to the arterial wall with circumferential blood flow at its distal most aspect, with a cyclical motion relating to the cardiac cycle".,3 Because of its rarity, the incidence among stroke patients is not well documented in the literature.

Chua (2012) identified this condition in twenty-five patients from a large carotid ultrasound database of 26,815 cases $(0.9 \%)$. Other studies report similar incidences of the condition. ${ }^{4,5}$

A floating thrombus is more frequently reported in male patients, with a male:female ratio of $2: 1^{1,4}$ The mean age of patients with floating thrombus is lower compared to patients affected by atherosclerotic carotid artery stenosis. The majority of reported patients are symptomatic, and most of the symptoms are due to distal arterio-arterial embolization, but a hemodynamic mechanism may also be involved. ${ }^{6}$

The exact etiology of a free-floating carotid thrombus is unknown, with published case series reporting different causes including complicated atherosclerotic plaques, hypercoagulability state, cardioembolism, dissection, traumatic vascular injury, aneurysm, thrombocytosis, and cocaine usage. ${ }^{1,4,6}$

Given its noninvasive nature, carotid duplex ultrasound examination is a useful diagnostic tool that can detect the majority of these lesions at the cervical level. ${ }^{2}$

Because of the low incidence of free-floating thrombus and the lack of evidence-based studies, the treatment methods are not standardized. Surgical and medical treatments have both been used, however there is no clear indication regarding the superiority of either. ${ }^{1,3}$
Hematological disorders are unusual causes of acute stroke. Ischemic stroke attributed to ET was found in $0.5 \%$ of acute cerebro-vascular disease cases. ${ }^{7}$ Thrombosis and hemorrhage are frequent complications of ET due to the qualitative and quantitative alterations of the thrombocytes. ${ }^{8}$

The thrombotic events secondary to ET are protean and include cerebral infarction, transient ischemic attacks, retinal artery occlusion, myocardial infarction, pulmonary embolism, portal vein thrombosis, deep venous thrombosis, and digital ischemia. ${ }^{9} \mathrm{ET}$, as a cause of ischemic stroke, is probably under-recognized, because it is usually not detected if the platelet count is not significantly elevated. ${ }^{7}$

There are no clear guidelines regarding the management of ischemic cerebro-vascular events secondary to ET. Antiplatelet drugs in combination with chemotherapeutic agents to control the thrombocytosis are usually recommended. ${ }^{7}$

The importance of MTHFR A1298C mutation in the current case was not evident.

\section{CONCLUSIONS}

A young patient with stroke required extensive imagistic and laboratory examination to establish an accurate etiological diagnosis. Carotid duplex ultrasound is an important part of this examination, as it can detect several vascular abnormalities such as atherosclerosis, thrombus and dissection.

In the current case two unusual findings, essential thrombocytosis and a free-floating carotid artery thrombus were observed and considered a direct cause of the repetitive transient ischemic attacks. Both conditions are rare. As there are no clear therapeutic guidelines in place, the treatment strategies must be individualized.

\section{ACKNOWLEDGEMENT}

This publication was supported by the Internal Research Grant of the University of Medicine and Pharmacy of Tîrgu Mureş, 26/ 11.12.2013.

\section{REFERENCES}

1. Bhatti AF, Leon LR Jr, Labropoulos N, et al. Free-floating thrombus of the carotid artery: literature review and case reports. J Vasc Surg. 2007;45:199-205. doi: 10.1016/j. jvs.2006.09.057.

2. Bajko Z, Balasa R, Petrutiu, S, Toma L, Russu S, Pascu I. Mobile carotid artery thrombus. Romanian Journal of Neurology. 2009;8:188-193. 
3. Elijovich L, Mainali S, Doss V, Arthur A, Watridge C. Medical management of free-floating carotid thrombus. Clin Neurol Neurosurg. 2013;115:1532-1535. doi: 10.1016/j. clineuro.2012.12.017.

4. Chua HC, Lim T, Teo BC, Phua Z, Eng J. Free-floating thrombus of the carotid artery detected on carotid ultrasound in patients with cerebral infarcts: a 10-year study. Annals Academy of Medicine Singapore. 2012;41:420-424.

5. Inatomi $\mathrm{Y}$, Mori A, Yonehara T, Hashimoto $\mathrm{Y}$, Hirano T, Uchino M. [Mobile thrombi in the cervical carotid artery]. Rinsho Shinkeigaku. 2005;45:711-716.

6. Csobay-Novák C, Járányi Z, Dósa E, Hüttl K. Asymptomatic free-floating thrombus of the internal carotid artery. Interventional Medicine \& Applied Science. 2011;3:213-215. doi: 10.1556/IMAS.3.2011.4.6.
7. Arboix A, Besses C. Cerebrovascular disease as the initial clinical presentation of haematological disorders. Eur Neurol. 1997;37:207-11.

8. Elliott MA, Tefferi A. Thrombosis and haemorrhage in polycythaemia vera and essential thrombocythaemia. Br J Haematol. 2005;128:275-290. doi: 10.1111/j.13652141.2004.05277.x

9. Polycythemia vera: the natural history of 1213 patients followed for 20 years. Gruppo Italiano Studio Policitemia. Ann Intern Med. 1995;123:656-664.

10. Kang S, Wu Y, Liu L, Zhao X, Zhang D. Association of the A1298C polymorphism in MTHFR gene with ischemic stroke. J Clin Neurosci. 2014;21:198-202. doi: 10.1016/j. jocn.2013.04.017. 